\title{
Strains of Pseudomonas syringae pv. syringae from Pea Are Phylogenetically and Pathogenically Diverse
}

\author{
Alberto Martín-Sanz, Marcelino Pérez de la Vega, Jesús Murillo, and Constantino Caminero
}

First and fourth authors: Instituto Tecnológico Agrario, Consejería de Agricultura y Ganadería de la Junta de Castilla y León, Ctra. Burgos, km 119, 47071 Valladolid, Spain; second author: Área de Genética, Departamento de Biología Molecular, Universidad de León, 24071 León, Spain; and third author: Departamento de Producción Agraria, ETS Ingenieros Agrónomos, Universidad Pública de Navarra, 31006 Pamplona, Spain.

Current address of A. Martín-Sanz: Laboratory of Plant Breeding, Graduate School for Experimental Plant Sciences, Wageningen University, Droevendaalsesteeg 1, 6708 PB Wageningen, The Netherlands.

Accepted for publication 25 January 2013.

\begin{abstract}
Martín-Sanz, A., Pérez de la Vega, M., Murillo, J., and Caminero, C. 2013. Strains of Pseudomonas syringae pv. syringae from pea are phylogenetically and pathogenically diverse. Phytopathology 103:673-681.

Pseudomonas syringae pv. syringae causes extensive yield losses in the pea crop worldwide, although there is little information on its host specialization and its interactions with pea. A collection of 88 putative $P$. syringae pv. syringae strains (including 39 strains isolated from pea) was characterized by repetitive polymerase chain reaction (rep-PCR), multilocus sequence typing (MLST), and $s y r B$ amplification and evaluated for

legume species. MLST data included all strains from pea in the genomospecies 1 of $P$. syringae pathovars defined in previous studies; they were distributed in the same three groups defined by rep-PCR. The inoculations performed in two pea cultivars showed that $P$. syringae pv. syringae strains from groups $1 \mathrm{~A}$ and $1 \mathrm{C}$ were less virulent than strains from group $1 \mathrm{~B}$, suggesting a possible pathogenic specialization in this group. This study shows the existence of genetically and pathogenically distinct $P$. syringae pv. syringae strain groups from pea, which will be useful for the diagnostic and epidemiology of this pathogen and for disease resistance breeding.
\end{abstract} pathogenicity and virulence. rep-PCR data grouped the strains from pea into two groups (1B and $1 \mathrm{C}$ ) together with strains from other hosts; a third group $(1 \mathrm{~A})$ was formed exclusively with strains isolated from non-
Additional keywords: pea bacterial blight, Pisum sativum.
Pseudomonas syringae can be found both as a harmless commensal on leaves and as a plant pathogen, causing disease (mostly foliar necroses) in practically every cultivated plant species (44). P. syringae has traditionally been divided into at least 60 pathovars, which are characterized by their host range $(6,8,44)$; as a whole, the species has an extremely large host range, while individual pathovars or strains are typically restricted to one or a small number of host species. P. syringae is a highly heterogeneous species, and DNA-DNA hybridization experiments allowed its reclassification, together with a few other Pseudomonas spp., into at least nine different genomospecies (12), which can also be differentiated by multilocus sequence typing (MLST) $(5,30)$. This heterogeneous group of bacteria is commonly known as $P$. syringae sensu lato $(12,44)$. Evolutionary genetic analyses reveal that, with respect to the core genome, $P$. syringae is a highly clonal and stable species that is endemic within plant populations, yet the genetic variation seen in these genes only weakly predicts host association (35).

Pea (Pisum sativum L.) bacterial blight can be caused by both Pseudomonas syringae pv. pisi and P. syringae pv. syringae (18). Both pathovars induce identical symptoms in the field and belong to the same genomospecies (genomospecies 1) (12); additionally, MLST analyses indicate that $P$. syringae pv. pisi can be separated into two phylogenetic groups, group 1 and group 2, of which

Corresponding author: C. Caminero; E-mail address: camsalco@itacyl.es

*The $\boldsymbol{e}$-Xtra logo stands for "electronic extra" and indicates that the online version contains three supplemental figures and two supplemental tables.

http://dx.doi.org/10.1094/PHYTO-08-12-0196-R

(c) 2013 The American Phytopathological Society group 1 clusters with strains of $P$. syringae pv. syringae from diverse hosts and other pathovars from genomospecies $1(27,34)$. However, there are no comprehensive studies that evaluate the biological characteristics and phylogenetic relationships of $P$. syringae pv. syringae strains isolated from pea; therefore, it is not clear whether or not these populations represent a homogeneous group of host-specialized strains or, alternatively, if pea is a general plant host for this pathogen. P. syringae pv. pisi is considered the main causal agent of bacterial blight of field pea and the most damaging, being able to cause disease over a wider range of environmental conditions than $P$. syringae pv. syringae (14). However, $P$. syringae pv. syringae can be as frequent and damaging as $P$. syringae pv. pisi in regions with frost risk when the crop is at seedling stage and under certain circumstances, especially in early sowings $(24,33)$. Disease symptoms are more evident after frost periods, heavy rains, or mechanical damage to the crop, all of which cause plant injuries that facilitate entry of the pathogen and infection initiation. Disease usually starts with the appearance of small water-soaked areas on any aerial plant part that, favored by humid and warm conditions, extend and eventually can lead to plant wilting and death (18). Control of pea bacterial blight is difficult and is mainly based on cultivation of resistant cultivars, crop rotation, and, because the pathogen is transmitted by seed, the use of pathogen-free seed (14). The use of pea cultivars combining bacterial blight resistance and frost tolerance has been described as a useful preventive control strategy (26).

$P$. syringae pv. syringae is a heterogeneous pathovar whose members are collectively able to cause disease in $>200$ different plant species $(4,17)$. It was described by Bradbury (4) as "a complicated mosaic of slightly different taxa from a large number of different host plants". One characteristic in common for most 
of the $P$. syringae pv. syringae strains is the production of toxic lipodepsinonapeptide metabolites, such as syringomycin (38). However, there is a certain degree of host specialization within this pathovar and there are strains classified as $P$. syringae pv. syringae that are not pathogenic to lilac or lemon fruit, which are considered as common hosts for strains of pv. syringae (37). P. syringae pv. syringae as a causal agent of pea bacterial blight was first described in 1966 in Wisconsin (13). The information for this taxon as a pea pathogen is still scarce, and pathogenic races have not been differentiated. In fact, there have been limited studies in relation to the presence or absence of host plant resistance, the genetic diversity of $P$. syringae pv. syringae strains isolated from pea, or their pathogenic variability and virulence. The knowledge of the structure and diversity of populations of this pathogen within the different countries is crucial for epidemiological studies, to define quarantine risks posed by exotic strains, and to facilitate the design of efficient strategies for pea bacterial blight control, including disease resistance breeding.

Several culture media as well as chemotaxonomic, metabolic, and molecular tests have been used to ascertain the relationships within $P$. syringae sensu lato (44). Biochemical tests have been traditionally used to differentiate pathovars of $P$. syringae and to evaluate inter- and intrapathovar variability (36). However, such tests cannot accurately discriminate between strains of $P$. syringae pvs. pisi and syringae pathogenic to pea (24). Diverse polymerase chain reaction (PCR)-generated genetic markers have also been used to distinguish these two pathovars. The most useful are a marker derived from the $s y r B$ gene, which is present in almost all the $P$. syringae pv. syringae strains $(24,37)$, and the AN3 and AN7 markers, found in repulsion in all the $P$. syringae pv. pisi strains analyzed $(2,24,27)$. Repetitive DNA PCR-based genomic fingerprinting (rep-PCR) with repetitive extragenic palindromic (REP), enterobacterial repetitive intergenic consensus (ERIC), and BOX primer sets is a sensible, reproducible, and highly discriminatory methodology to assess bacterial diversity at the strain and pathovar level $(21,22)$. Several studies have successfully used rep-PCR for genetic characterization of different pathovars of $P$. syringae $(10,26,29,37)$. On the other hand, MLST focuses strictly on the bacterial core genome. This highly accurate and reproducible approach uses the DNA sequences from housekeeping genes to differentiate strains and clonal lineages. MLST has been used to study genetic diversity and evolution in $P$. syringae sensu lato and to identify pathovars and strains within this species $(5,10,16$, 26,35,39).

The objectives of this study were to (i) assess the genetic and pathogenic diversity of a collection of $P$. syringae pv. syringae strains from different countries and hosts, including a representative set of strains isolated from pea; (ii) investigate the relationships between $P$. syringae pv. pisi and $P$. syringae pv. syringae strains isolated from pea, and also in relation to other pathovars and $P$. syringae pv. syringae strains isolated from different host species; (iii) analyze the relationship between the genetic patterns and the virulence of the strains in two pea cultivars; and (iv) clarify whether the strains isolated from pea in Spain originate from one or several inoculum sources.

\section{MATERIALS AND METHODS}

Bacterial strains. A collection of 88 putative Pseudomonas syringae pv. syringae isolates and strains was used, including 29 isolates obtained from infected fields in the main pea-producing area of Spain (24) and 10 strains isolated from pea in other countries. The remaining 49 strains were included as a representation of the diversity of this pathovar, including the pathotype strain (CFBP 1392, the same as LMG 1247). The strains and isolates used in this study include a representation of 19 countries and 30 hosts (both woody and herbaceous host plants), and they are described in detail in Supplemental Table 1. Bacteria were routinely grown on King's B medium $(\mathrm{KB})$ plates and preserved in media with $15 \%$ glycerol at $-75^{\circ} \mathrm{C}$ for long-term storage. For simplicity, all strains from collections and Spanish isolates will be hereafter referred as strains.

Strain identification. Strains were identified according to a classical methodology (36), including grouping by gram reaction using the $\mathrm{KOH}$ test; Hugh-Leifson oxidation/fermentation test; fluorescence on KB medium; levan production, oxidase reaction, potato soft rot, arginine dihydrolase activity, and tobacco hypersensitivity (LOPAT) test (19); the use of homoserine, betaine, D-tartrate, erythritol, and trigonelline as carbon sources; and aesculin and gelatine hydrolysis. Duplicate tubes or plates were used for each test, and tests were repeated at least twice.

The AN3 (132 bp) and AN7 (272 bp) markers and the $s y r B$ gene marker (752 bp) were PCR amplified using specific primer pairs $(2,38)$ and DNA from well-isolated colonies as template. Each amplification reaction was carried out at least twice. Negative controls (sterile deionized water) and positive controls ( $P$. syringae pv. syringae pathotype strain CFBP 1392 for syrB; type strains of race 1, HRI-W 299A, and race 2, HRI-W 202, of $P$. syringae pv. pisi for AN3 and AN7, respectively) were included. Gene $\operatorname{syr} B$, whose product is involved in the production of cyclic lipodepsinonapeptides, is present in $P$. syringae pvs. atrofaciens, aptata, and syringae but not in others such as $P$. syringae pvs. pisi or phaseolicola. AN3 and AN7 markers are specific to $P$. syringae pv. pisi.

rep-PCR. Strains were grown on $\mathrm{KB}$ plates at $25^{\circ} \mathrm{C}$ for $48 \mathrm{~h}$ and genomic DNA was purified using the Qiagen DNeasy Tissue Kit following the manufacturer's instructions. The concentration of DNA was estimated by electrophoresis of $2 \mu$ of DNA suspensions, together with a DNA marker, on a $1 \%$ agarose gel with $1 \times$ Tris-acetate-EDTA (TAE) buffer and stained with ethidium bromide. Total genomic DNA was adjusted to a final concentration of $20 \mathrm{ng} / \mu \mathrm{l}$ and stored at $-20^{\circ} \mathrm{C}$ before use. rep-PCR with REP-PCR, ERIC-PCR, and BOX-PCR primers was performed as described previously (32). PCR products were separated by electrophoresis in $1.5 \%$ agarose gels in $0.5 \times$ TAE and visualized under UV light after staining with ethidium bromide. Strains were tested at least three times with each rep-PCR method to ensure reproducibility of the DNA fingerprints.

MLST. MLST was performed with partial sequences of gapl, gyr $B$, and $r p o D$ genes coding for glyceraldehyde-3-phosphate dehydrogenase, DNA gyrase B, and $\sigma$ factor 70 , respectively. Gene fragments were amplified from genomic DNA of $16 P$. syringae pv. syringae strains, which represented the rep-PCR variability of the collection, with the primers described by Sarkar and Guttman (35) and according to the PCR procedure described previously (10). PCR products were purified with the QIAquick PCR purification kit (Qiagen, Hilden) following the manufacturer's instructions and sequenced using a Megabace 500 (Amersham, Freiburg) sequencer. Sequences were edited, aligned, and compared with other Pseudomonas sp. sequences (35) obtained from the National Center for Biotechnology Information database and the PlantAssociated Microbes Database (1) (Supplemental Table 2) by using the MEGA5 program (41). Additional $P$. syringae sequences were selected to include other groups of strains and genomospecies from $P$. syringae sensu lato $(5,35)$. Furthermore, $P$. syringae pv. pisi sequences described in previous research $(27,35)$ were included for comparison of the two main $P$. syringae pathovars that infect pea.

Data analysis. The size of the fragments amplified by rep-PCR was estimated using a DNA 100-bp size-ladder (DNA Molecular Weight Marker XIV; Roche, Mannheim). Band presence or absence was scored as a 1 or 0 , respectively. Only clear and repeatable (in three replicas) bands were scored. The strains were grouped in dendrograms using distance derived from Jaccard similarity indices. Distances were calculated as 1-similarity. Unweighted pair-group method with arithmetic means (UPGMA) 
and neighbor-joining $(\mathrm{NJ})$ clustering methods were used to obtain dendrograms by means of the PHYLIP 3.63 package (9), and confidence was estimated by bootstrap with 1,000 resamplings. Population structure was analyzed by means of the Structure program 2.3 (31). For MLST analysis a concatenated data set (1,181 aligned nucleotides) was used to construct dendrograms in MEGA5 using the Tamura 3-Parameter distance (40) and the NJ method to infer the genetic relationships between $P$. syringae strains; confidence levels of the branching points were determined using 1,000 bootstrap replicates. $P$. syringae pv. pisi strains with identical concatenated sequences were represented by a single sequence in the dendrogram analyses. The MLST sequences generated were deposited in the EMBL GenBank database with the following accession numbers: gapA, HE604351 to HE604365 and HE565700; gyrB, HE604381 to HE604395 and HE565702; and rpoD, HE604396 to HE604410 and HE565703. These sequences were also submitted to the Plant Associated and Environmental Microbes Database (1).

Pathogenicity tests. Bacterial strains were inoculated on immature lemon fruit (Citrus lemon 'Primofioro'), excised bean pods (Phaseolus vulgaris 'Ancha Lisa'), and excised pea pods (Pisum sativum 'Ucero') according to a previous methodology (37) to assess their host range. Plant material was first surface sterilized with $70 \%$ ethanol and rinsed in distilled sterilized water; then, strains were inoculated by depositing $20 \mu \mathrm{l}$ of a bacterial suspension $\left(10^{8} \mathrm{CFU} / \mathrm{ml}\right)$ onto punctures made with sterilized entomological needles at 10 sites per strain. Ten sites within two fruit or pods were inoculated with each strain. Inoculated lemon fruit, bean pods, and pea pods were kept in a humid chamber at $20^{\circ} \mathrm{C}$ for 7 days and the infection response was recorded daily, scoring the presence (water-soaked, necrotic lesions, and so on) or absence of symptoms. Positive controls inoculated with strains P684 (Pseudomonas syringae pv. phaseolicola race 7; for bean pods), HRI-W 895A ( $P$. syringae pv. pisi type race 4; for pea pods), and CFBP 1769 (P. syringae pv. syringae; for lemon fruit) were included.

Subsequently, bacterial strains were inoculated in the stem of 2-week-old seedlings of 'Gracia' and 'Iceberg' pea, susceptible and resistant, respectively, to $P$. syringae pv. syringae $(24,26)$, to evaluate their virulence. The inoculation methodology and growing conditions of pea seedlings under controlled conditions were essentially as described previously (26). Stem inoculations were carried out by scraping freshly grown bacteria from the surface of $\mathrm{KB}$ plates with the tip of a sterile entomological mounting pin and then stabbing it into the main stem at its junction with the stipules at the youngest node. Nine seedlings per pea cultivar were inoculated with each bacterial strain in three independent replicates (three plants per replica). The response was scored 10 days after inoculation using an arbitrary scale, as follows: 0, small localized necrosis in the infection point; 1, necrotic spots around the infection point not longer than $0.5 \mathrm{~cm}$; 2, necrotic lesions of 0.5 to $1.5 \mathrm{~cm} ; 3$, lesions longer than $1.5 \mathrm{~cm}$; and 4, wilting of the seedlings from the inoculation point to the apex; an example of these reactions is shown in Supplemental Figure 3. Strains were classified into five arbitrary virulence categories depending on the averaged values for each cultivar-strain combination, as follows: 0 to 0.5 , nonpathogenic (NP); 0.6 to 1.5 , weakly virulent $(\mathrm{W}) ; 1.6$ to 2.5 , moderately virulent $(\mathrm{M}) ; 2.6$ to 3.5 , highly virulent $(\mathrm{H})$; and 3.6 to 4 , extremely virulent, causing apical death $(\mathrm{AD})$.

TABLE 1. Pathogenic and metabolic variability among and within the groups established by repetitive polymerase chain reaction (rep-PCR) and multilocus sequence typing (MLST) for Pseudomonas syringae pv. syringae strains isolated from pea and other hosts

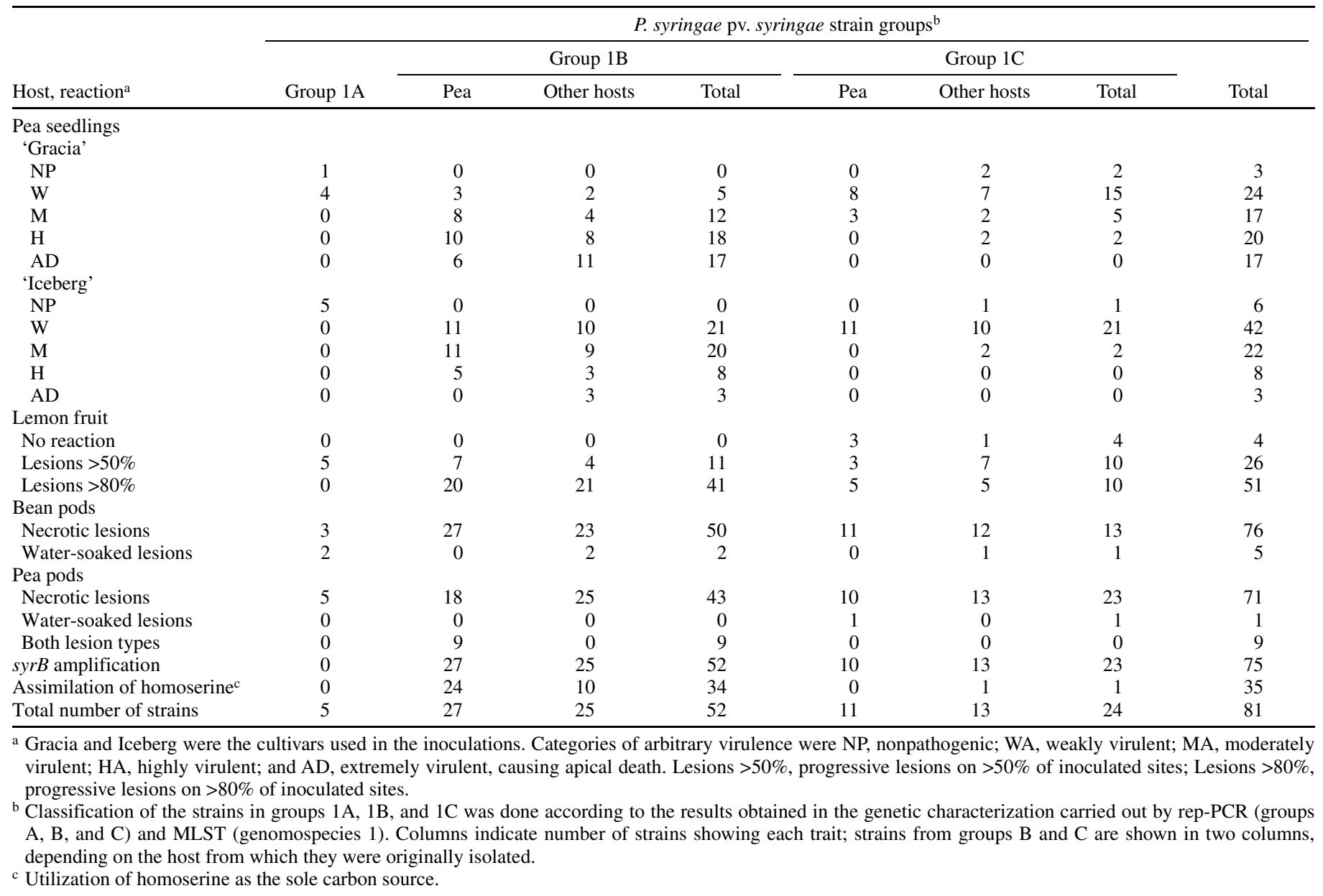




\section{RESULTS}

Identification and characterization of bacterial strains. The 88 strains analyzed in this study were gram-negative, oxidative in Hugh-Leifson medium, positive for levan production and induction of the hypersensitivity response on tobacco leaves, and negative for the presence of oxidase and arginine dihydrolase and for potato rotting (LOPAT IA). Moreover, the majority of the strains (>90\%) hydrolyzed aesculin; liquefied gelatin; utilized Dtartrate, trigonelline, and erythritol; and were fluorescent on $\mathrm{KB}$ medium. There was variability in the assimilation of homoserine

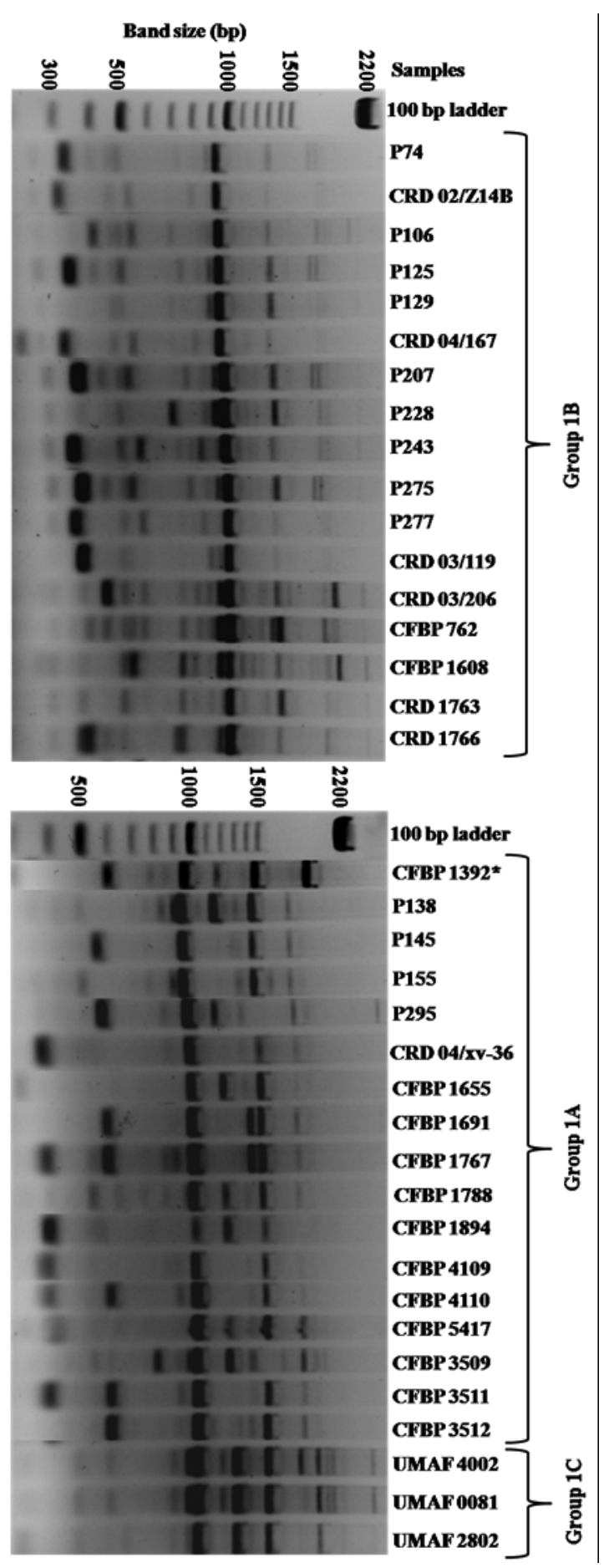

Fig. 1. Representative repetitive polymerase chain reaction (rep-PCR) patterns of Pseudomonas syringae pv. syringae strains performed by repetitive extragenic palindromic-PCR. A 100-bp ladder is included; * indicates pathotype strain.
(42\% of positive responses) and betaine (59\%). Most of the strains that could utilize homoserine as the sole carbon source were isolated from pea (from Spain, New Zealand, and Australia) and common vetch (Vicia sativa) and Lathyrus spp. (Spain). Finally, in total, 75 strains amplified the 752-bp fragment of the syrB gene and none of them amplified the specific markers for P. syringae pv. pisi (AN3 and AN7).

Inoculation of lemon fruit, bean and pea pods, and pea seedlings showed that 81 strains were pathogenic to at least one of these plant hosts. The inoculation in lemon fruit showed that 77 strains $(87.5 \%$ of the total) were pathogenic: 26 strains were classified as + (progressive lesions on more than $50 \%$ of inoculated sites), and 51 were ++ (progressive lesions on more than $80 \%$ of inoculated sites). The most common response observed in the inoculation of bean pods was progressive necrosis (76 strains, $86.4 \%$ of the total), followed by nonreaction ( 7 strains, $7.9 \%$ ) and water-soaking ( 5 strains, $5.7 \%$ ). In the case of the pea pods, the main response was progressive necrosis (71 strains, $80.7 \%$ ), then a mixture of water-soaking and necrotic symptoms (9 strains, $10.2 \%)$, nonpathogenic reaction (7 strains, $7.9 \%$ ), and watersoaking (1 strain, 1.1\%). Seven strains (CFBP 2337, CFBP 6886, NCPPB 2747, NCPPB 2805, NCPPB 3163, NCPPB 3185, and NCPPB 3259) did not amplify $s y r B$ and were nonpathogenic on lemon fruit, pea and bean pods, and pea seedlings. For this reason, they were not considered to be $P$. syringae pv. syringae and not included in further analysis. A summary of the results of some tests for $P$. syringae pv. syringae strains isolated from pea and other host species is shown in Table 1. The individual response of the strains to all these tests (biochemical tests, inoculations, and $s y r B$ amplification) is shown in Supplemental Table 1.

rep-PCR. The rep-PCR analyses yielded a total of 91 clearly observable polymorphic bands of 0.1 to $2.2 \mathrm{~kb}$ (Fig. 1). Strains showed a high level of heterogeneity, generating 54 different repPCR patterns. REP-, ERIC-, and BOX-PCR generated 31, 34, and 26 polymorphic bands, respectively. The NJ and UPGMA dendrograms elaborated individually with BOX-, REP-, or ERICPCR bands and, with all the rep-PCR polymorphic bands, showed consistent trees with a similar topology (Fig. 2; Supplemental Figure 1) but with low bootstrap values. According to these dendrograms and the Structure analysis (Supplemental Figure 2), the strains could be grouped into three main clusters. They have been called as groups $1 \mathrm{~A}, 1 \mathrm{~B}$, and $1 \mathrm{C}$, where the letters differentiate the three clusters and the number refers to genomospecies 1. Group $1 \mathrm{~A}$ is formed by only five strains from three countries (Spain, Australia, and Brazil), and isolated from three hosts (Solanum lycopersicum, Mangifera indica, and Piper nigrum). Group 1B contains 52 strains, which is the majority of the strains evaluated in this study, having a representation of 15 host species from 10 countries. It is worth mentioning that this group includes 27 strains isolated from pea from South Africa (1 strain), Australia (2 strains), New Zealand (3 strains), and Spain (21 strains). Finally, group $1 \mathrm{C}$ includes 24 strains from 10 hosts and 10 countries. In total, 11 strains from this group were isolated from pea (8 in Spain, 2 in the United Kingdom, and 1 in France). The pathotype strain CFBP 1392 is located in this cluster. The rep-PCR fingerprints of the nonpathogenic and $s y r B$-negative strains (CFBP 2337, CFBP 6886, NCPPB 2747, NCPPB 2805, NCPPB 3163, NCPPB 3185 [isolated from pea], and NCPPB 3259), whose inclusion in $P$. syringae pv. syringae was described previously as questionable, were very different from those observed for the remaining strains. These strains were not included in the dendrograms generated.

This assay revealed genetic variability among strains collected from the same plant species. This is the case for the 2 strains isolated from Syringa vulgaris (CFBP 1392 and CFBP 1766, placed in groups $1 \mathrm{C}$ and $1 \mathrm{~B}$, respectively) and the strains from pea, with 11 located in group $1 \mathrm{C}$ and 27 in group 1B. In all, the 38 pathogenic Pseudomonas syringae pv. syringae strains isolated from 
pea represent 21 rep-PCR patterns: 11 placed in group $1 \mathrm{C}$ and 10 in group $1 \mathrm{~B}$.

MLST analysis. A subset of 16 strains (10 of them isolated from pea), selected to cover the genetic diversity revealed by repPCR, was analyzed by MLST (1,181 aligned nucleotides from three partial gene sequences). Each of these strains showed a different MLST sequence profile. This analysis also clearly discriminated the three groups identified by rep-PCR, and confirmed the existence of a high genetic variability among $P$. syringae pv. syringae strains isolated from pea (Fig. 3). Subsequently, in order to study the relation of the $P$. syringae pv. syringae strains used in this work with $P$. syringae pv. pisi and other $P$. syringae strains, a MLST dendrogram was also generated with the sequences from those $16 P$. syringae pv. syringae strains plus $P$. syringae pv. pisi strains from Martín-Sanz et al. (27), other $P$. syringae strains, and pathovars employed in previous studies $(5,35)$ (Fig. 3). All P. syringae pvs. syringae and pisi strains were grouped within a cluster which agreed with genomospecies 1 and the related genomospecies 7 (12), except the strain Ps 9220. Additionally, the $P$. syringae pv. syringae strains could be separated into three subclusters, supported by high bootstrap values, which are in agreement with the groups defined previously by rep-PCR (Fig. 2). Subcluster A included two $P$. syringae pv. syringae strains clearly discriminated from all other $P$. syringae pvs. syringae and pisi strains in genomospecies 1 ; subcluster B was formed exclusively by $P$. syringae pv. syringae strains, all of them except one isolated from legume crops; while subcluster $\mathrm{C}$ included all the $P$. syringae pv. pisi strains and some $P$. syringae pv. syringae strains. Within subcluster $\mathrm{C}, P$. syringae pv. pisi sequences are clearly differentiated into two sets, one including nine strains with the sequence designated Ppi 1 (see strain names in Figure 3 caption) and closely similar sequences, all of which amplified the AN7 marker, and a second one with five strains with sequences designated Ppi 2 (Fig. 3), which amplified the AN3 marker and had an MLST nucleotide sequence identical to that of strain $P$. syringae pv. syringae $\mathrm{P} 285$, also isolated from pea.

Pathogenicity on pea cultivars. A classification of the strains used in this work according to their pathogenic responses in the two tester pea cultivars is shown in Table 1. Stem inoculation of Gracia and Iceberg pea showed that strain NCPPB 2264 and CFBP 1679 were not pathogenic on pea seedlings. Likewise, strain CFBP 1894 was not pathogenic to Gracia but, in contrast was pathogenic to Iceberg. Conversely, strains CFBP 1789, UMAF 2802, UMAF 4002, and UMAF 0081 were pathogenic to Gracia but not to Iceberg. The remaining strains were pathogenic, producing brown necrotic lesions in the inoculation points that moved upward to the apex, sometimes producing AD. There was an important variability in the length of the lesions depending upon the combination of $P$. syringae pv. syringae strain-pea cultivar evaluated. The virulence spectrum of the $P$. syringae pv. syringae strain collection evaluated in this work is visually represented in Supplemental Figure 3, whereas the response elicited by each strain upon stem inoculation in both pea cultivars is shown in Supplemental Table 1. First, it is clear that the strain collection behaved more aggressively in Gracia than in Iceberg, as was expected, because the first pea genotype was more susceptible to $P$. syringae pv. syringae in other studies $(24,26)$. Second, it is interesting that the virulence of the strains toward pea is also correlated with their grouping by rep-PCR and MLST analyses. Thus, strains from groups $1 \mathrm{C}$ and $1 \mathrm{~A}$ seem to be much less aggressive than strains from group 1B; however, a wider strain sample would be needed to confirm this result. This trend was

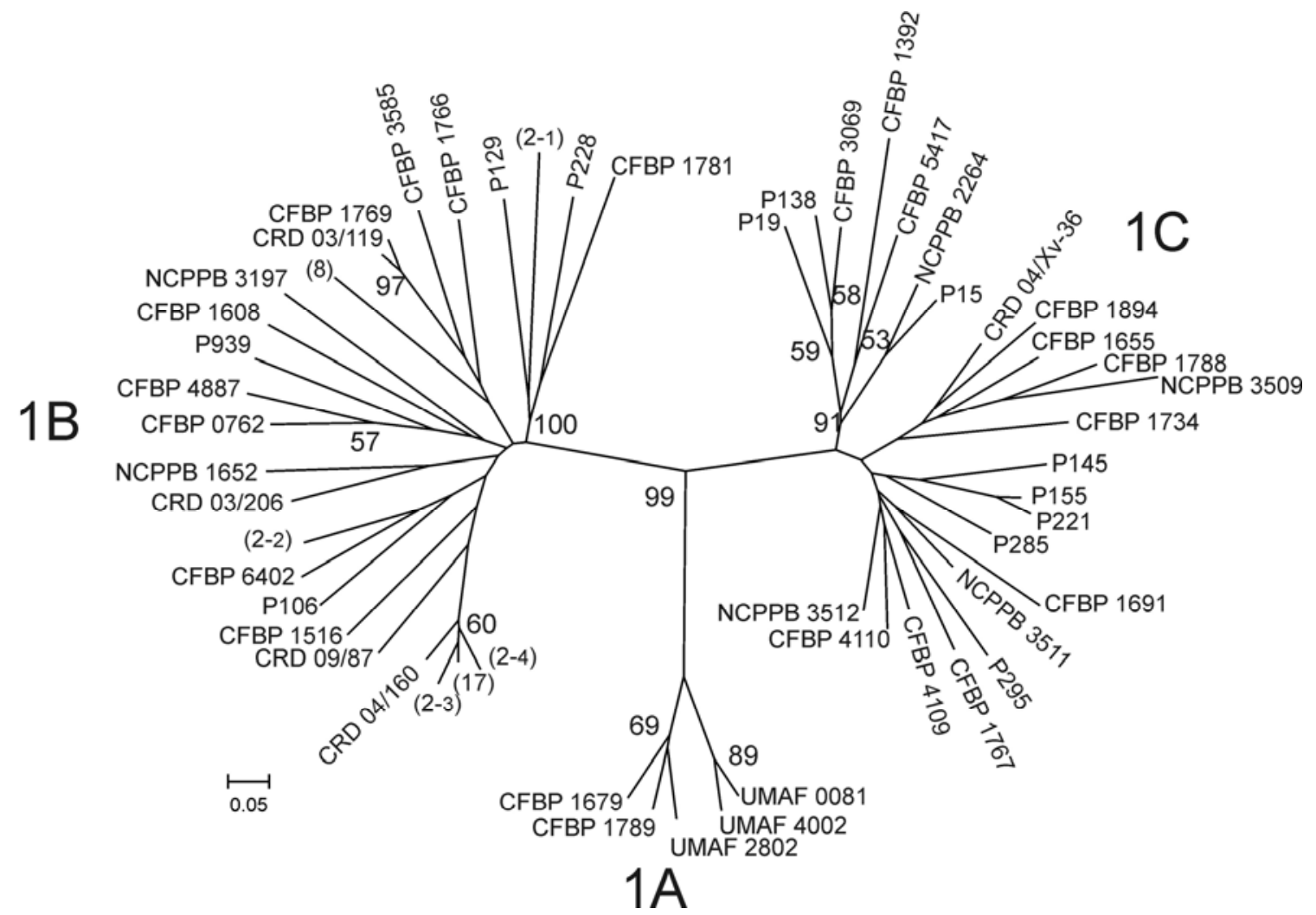

Fig. 2. Neighbor-joining tree of 81 Pseudomonas syringae pv. syringae strains based on the combined repetitive polymerase chain reaction (rep-PCR) data. Figures indicate the bootstrap supporting values $>50$; figures within parentheses indicate the number of strains with the same rep-PCR pattern included in a given branch (2-1 = CFBP 1763 and CFBP 1768; 2-2 = UMAF 6016 and UMAF 6582; 2-3 = NCPPB 3505 and NCPPB 3506; 2-4 = P59 and CRD 02/Z14B; $8=$ P74, P125, P207, P275, P487, P488, P526, and P528; 17 = CRD 02/231, CRD 03/199, CRD 04/167, NCPPB 2688, NCPPB 2689, P144, P151, P185, P204, P243, P262, P271, P277, P522, P523, P524, and P525). Each set of repeated rep-PCR patterns was computed as a single pattern. The nomenclature was chosen to coincide with the multilocus sequence typing groups defined for strains of genomospecies 1 (Fig. 3) and, for this reason, the three groups detected by rep-PCR are named $1 \mathrm{~A}, 1 \mathrm{~B}$, and $1 \mathrm{C}$. 
also observed when only strains isolated from pea were considered. Finally, it is worth mentioning that the most aggressive strains (AD response in Gracia and $\mathrm{AD} / \mathrm{H}$ in Iceberg) are those isolated not only from pea but also from other hosts such as common lilac, common bean, hairy vetch, and melon.

\section{DISCUSSION}

rep-PCR has been widely applied to classify and analyze the genetic differentiation of species within bacterial genera $(21,22)$ and has been useful to assess the diversity of $P$. syringae patho-

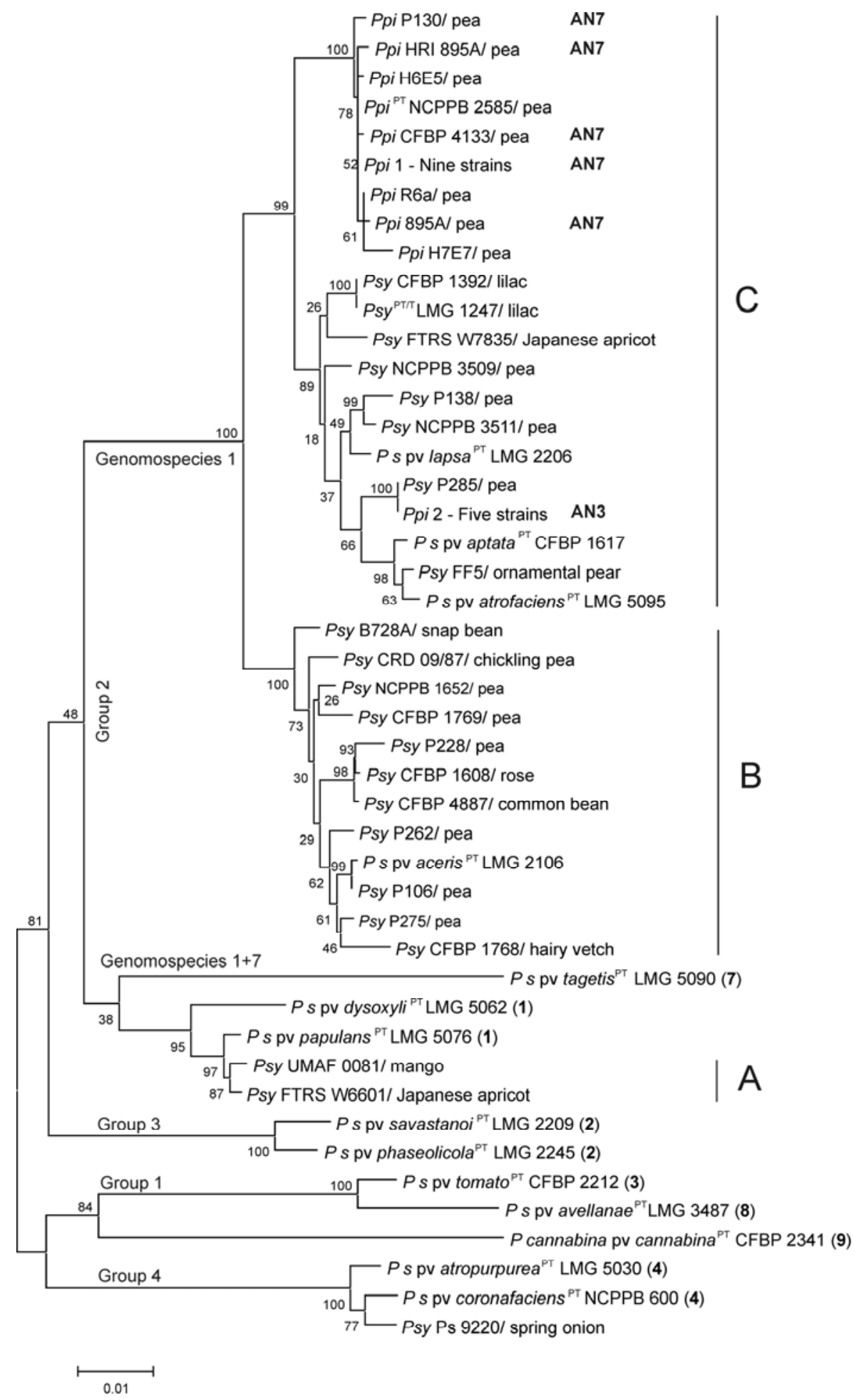

Fig. 3. Neighbor-joining tree of the Pseudomonas spp. multilocus sequence typing concatenated data set (1,181 bp). Pseudomonas syringae pvs. syringae and pisi are abbreviated as Psy and Ppi, respectively, and the host of isolation is given next to each strain designation. The four groups and the genomospecies refer to those defined by Sarkar and Guttman (35), Bull et al. (5), and Gardan et al. (12). P. syringae pv. pisi strains with identical concatenated sequences are represented by a single sequence, as follows: Ppi 1 corresponds to P. syringae pv. pisi strains H5E1, P123, P134, P282, CFBP 4767, CFBP 4485, HRI-W 202, HRI-W 870A, and HRI-W 1704B; Ppi 2 corresponds to P. syringae pv. pisi strainsH5E3, P68, CFBP 4746, CFBP 4484, and HRI-W 299A. Vertical lines on the right indicated the three subgroups described within group 2. AN3 and AN7 indicate the specific marker amplified by each set of $P$. syringae pv. pisi strains. Type and pathotype strains are indicated by T or PT, respectively. Bold numbers within parenthesis on the right indicated the genomospecies of pathovars other than syringae and pisi. Bootstrap node support values are indicated. More detailed strain identification is shown in Supplemental Table 2. 
vars $(10,27,29,37)$. In the present study, rep-PCR allowed differentiating 54 genetic patterns that clustered 81 P. syringae pv. syringae strains into three groups (1A, 1B, and 1C) on NJ (Fig. 2) and UPGMA dendrograms and Structure analysis. P. syringae pv. syringae strains isolated from pea clustered in group 1B (27 strains) and 1C (11 strains), showing a relatively high genetic diversity. However, this probably has a very limited significance because the large majority of the analyzed strains were pathogenic to pea independently of their host of isolation, reflecting the typical wide host range of $P$. syringae pv. syringae (4) and indicating that strains of this pathovar might survive in the environment, moving among compatible plant hosts. The very different rep-PCR fingerprints of the seven strains which were nonpathogenic to pea, lemon, or bean supports the possibility that they were misclassified as $P$. syringae pv. syringae; therefore, they were not included in further analyses.

The MLST analysis was mainly carried out using sequences from diverse strains isolated from field epidemics in Spain and sequences previously used in other phylogenetic analyses $(5,35,39)$. Our data agree with the general dendrogram topologies described in those works: the $P$. syringae pathovars analyzed in this work are distributed in four clades. All $P$. syringae pv. syringae strains are included in genomospecies 1 except strain Ps 9220 which, in our dendrogram and in previous ones $(16,35)$, is included in genomospecies 4 (Fig. 3). It is possible, therefore, that this strain does, indeed, belong to a different pathovar and has not been correctly identified. Likewise, $P$. syringae pv. syringae strains are distributed into three clusters (A, B, and C) within genomospecies 1 , and $P$. syringae pv. pisi strains are included in one of these clusters ( $\mathrm{C}$ in our case), splitting into two clearly different genetic lineages.

The MLST analysis also agrees with the rep-PCR results and supports the existence of at least three different genetic lineages in $P$. syringae pv. syringae. Most of the $P$. syringae pv. syringae strains from pea cluster in group B together with other strains of this pathovar isolated from other legume crops. The $P$. syringae pv. syringae strains from group $\mathrm{C}$ are the most similar to $P$. syringae pv. pisi but, although strains from this pathovar amplifying the AN7 marker are discriminated in a subgroup supported with a $100 \%$ bootstrap value (Fig. 3), our results showed a very high genetic similarity between $P$. syringae pv. syringae strains in cluster $\mathrm{C}$ and strains of $P$. syringae pv. pisi amplifying marker AN3. For instance, the MLST sequences from strain $P$. syringae pv. syringae P285 were identical to those from strains in group Ppi 2 (Fig. 3). Additionally, these sequences cluster very close to those from the recently sequenced strain $P$. syringae pv. syringae FF5, isolated from ornamental pear and the pathotype strains from pvs. aptata and atrofaciens, although these last three pathovars might be synonymous (44). Lack of correlation between genetic proximity and pathovar assignation has already been shown for pvs. avellanae, lachrymans, and morsprunorum, which contain strains belonging to two different genomospecies $(12,28)$, showing that evolutionary convergence of pathogenicity is somewhat frequent in $P$. syringae. However, the occurrence of two genetic lineages of $P$. syringae pv. pisi within genomospecies 1 seems to be due, instead, to two independent events of host specialization. Indeed, certain phytopathogenic $P$. syringae with a narrow host range and infecting current monoculture agricultural crops appear to have evolved from ancestors with a broader host range that were adapted to natural mixed plant communities (7). In support of our hypothesis, the widespread distribution of the ability to infect pea among the three $P$. syringae pv. syringae genetic lineages suggests that this pathogenic capability is ancestral to genomospecies 1. Remarkably, P. syringae pv. pisi 1704B (belonging to the AN7 group) has gained numerous type III effectors that are not present within the genomes of five strains from other pathovars of genomospecies $1(3,20)$, and at least two of these genes (hopCl and hopZ2) $(3,43)$ act negatively by pre- venting infection of bean plants, a common host for the three lineages of $P$. syringae pv. syringae (34). P. syringae pv. pisi and $P$. syringae pv. syringae shared 12 type III effector families (3) but there are 7 specific families for $P$. syringae pv. pisi and 3 for $P$. syringae pv. syringae. The effectors in common could act as general virulence factors in pea while the specific ones could play roles in the diversification of these two closed pathovars. Moreover, these two pathovars are able to produce different phytotoxins, with syringomycin, syringopeptin, and syringolin being exclusively produced by $P$. syringae pv. syringae $(20,42)$. Therefore, it is possible that a gain in effector genes by strains of $P$. syringae pv. pisi has resulted in a reduction in host range, effectively limiting the occurrence of this pathovar in agricultural ecosystems to the pea crop, although it is known that $P$. syringae pv. pisi can affect other plant hosts such as lentil (Lens culinaris L.) in controlled conditions, with which it displays a race structure (15). However, it is not known whether there has been a similar massive gain of effector genes in the $P$. syringae pv. pisi AN3 group.

Given the complex phylogenetic relationships among the diverse genetic lineages in pvs. pisi and syringae, is it justified that they are considered different pathovars? Pathovars are defined as a "...set of strains with the same or similar characteristics, differentiated at infrasubspecific level from other strains of the same species... on the basis of distinctive pathogenicity to one or more plant hosts" (9). Although strains of both pathovars produce indistinguishable symptoms in natural field infections, they induce distinctive symptoms in pea and other hosts in inoculations in controlled conditions: $P$. syringae pv. syringae produces necrotic symptoms on pea while $P$. syringae pv. pisi generates water-soaked lesions $(4,24,26,27,37)$. Likewise, $P$. syringae pv. pisi is a pathogen restricted to pea whereas $P$. syringae pv. syringae can cause disease in additional hosts. Therefore, we believe that their differential pathogenicity fully justifies the separation of these two pathovars.

The existence of different genetic lineages of $P$. syringae pv. syringae and $P$. syringae pv. pisi also significantly impact our ability to efficiently differentiate strains from both pathovars. The use of homoserine as the sole carbon source has been traditionally evaluated to distinguish between $P$. syringae pvs. syringae and pisi strains isolated from pea, because the first pathovar was described as unable to use this amino acid while the second was described as positive (36). However, at least several $P$. syringae pv. syringae strains utilized homoserine as the sole carbon source (24; this study). Thus, in total, 34 of the 52 strains included in group 1B $(65.4 \%$ of the total of this group and $88.9 \%$ of the strains from pea) and CFBP 4110, included in group 1C, were positive for this test. The positive strains included in group 1B were isolated from pea and other legume crops in several countries, although group $1 \mathrm{~B}$ also included strains unable to use homoserine isolated from pea in different countries. Therefore, these data indicate that the assimilation of homoserine is not a reliable test and should not be used as the only criterion to differentiate $P$. syringae pvs. syringae and pisi strains from pea. In fact, the ability to use homoserine seems to be associated with other genetic characteristics because all pv. syringae strains included in group A and 23 of the 24 in group 1C were negative, whereas most of them in B were positive. Thus, we can conclude that inoculation in pea and amplification of AN3/7 (amplified in repulsion from all $P$. syringae pv. pisi strains) and $s y r B$ (amplified from most $P$. syringae pv. syringae strains) markers are the most important criteria to differentiate between these two pathovars. Nevertheless, further studies are needed, including the identification of host-specific virulence factors, for the rapid and efficient differentiation of these two pathovars.

MLST and rep-PCR results support a possible linkage between genetic lineages and virulence in two pea cultivars. However, the degree of virulence toward two pea cultivars was not correlated 
with the host of isolation, with certain strains isolated from nonlegumes showing high levels of virulence to pea. According to the symptoms observed in both lemon fruit and pea seedlings (Table 1), strains in group 1A were the least aggressive and, incidentally, none of them produced a specific amplicon with the $s y r B$ primers, whereas those in group 1C showed an intermediate virulence. This trend was observed when considering all the strains or only strains isolated from pea. In turn, group 1B appears to contain a larger percentage of highly virulent strains than groups $1 \mathrm{~A}$ and $1 \mathrm{C}$, although this could be biased by the limited sample size of these two last groups. Additionally, it is possible that the degree of virulence against the two pea cultivars tested is not broadly representative of the intrinsic virulence potential of each strain. In support of this, a few strains show high virulence toward one of the cultivars but only weak virulence toward the other.

Knowledge about the genetic diversity of $P$. syringae pv. syringae strains from pea and other hosts and their virulence is pivotal for the selection of appropriate strains to be used in disease resistance testing. The high level of pathogenic and genetic variability found in the populations evaluated in the present study can provide a basis for selecting useful strains to identify and characterize resistance genes in pea. Very aggressive strains from group 1B are good candidates for resistance selection screening in pea germplasm collections and to study host specialization of this pathovar toward pea. Indeed, the highly virulent strain P262 has been used in the search of resistance to $P$. syringae pv. syringae in pea and in the genetic mapping of resistance genes $(11,26)$. P262 has the same rep-PCR genetic pattern as 12 other strains isolated from pea (10 from Spain and 2 from New Zealand) and 4 from chickling pea (Spain), and it is also very close to another 5 strains ( 3 isolated from pea in Spain and 2 in Australia). Considering these data and the information about the resistance to this pathovar in pea described by Martín-Sanz et al. (26), it is clear that future studies are needed to detect more significant pea cultivar- $P$. syringae pv. syringae strain interactions, and to know whether it is possible to differentiate pathogenic races, which will be very useful for disease resistance breeding and to provide a clearer picture of the pathogenic specialization of this pathogen.

It is not possible to establish with complete certainty the origin of the $P$. syringae pv. syringae strains collected from pea bacterial blight epidemics during the last 10 years in Spain, when growers started to use early (autumn-winter) sowing for the pea crop. However, these results suggest that seed batches contaminated with $P$. syringae pv. syringae contributed to the initiation or expansion of these epidemics, when the area devoted to pea increased considerably. This hypothesis is supported by the fact that Spanish strains present a genetic diversity that is comparable with that of strains from other countries, and because this seedborne pathogen was unknown as a pea pathogen in Spain until approximately the turn of the century. A similar hypothesis has been proposed for the origin of Spanish $P$. syringae pv. pisi isolates (27). Because $P$. syringae pv. syringae is a polyphagous pathogen, the possibility that cross contamination from other hosts could increase the genetic diversity of the Spanish $P$. syringae pv. syringae population in pea cannot be ruled out. This is supported by the fact that this pathogen was recently identified in other grain legumes crops such as common vetch, chickling pea, and grass pea in the same area of Spain $(23,25)$; because four of the five $P$. syringae pv. syringae strains from chickling pea have rep-PCR patterns identical to strains from pea; and because strains from common vetch and grass pea are identical to each other and to strains from pea isolated in Spain. All these data stress the need to increase the phytosanitary controls of seed lots and fields of all these crop species to avoid the expansion of the pathogen.

Results from this work provide the foundations for a better understanding of the genetic structure and diversity among strains of $P$. syringae pv. syringae; in particular, those isolated from pea.
It is clear that there are genetically and pathogenically distinct strain groups isolated from pea. Furthermore, this study also contributes to further our knowledge on the relationships between the two most important pea bacterial pathogens ( $P$. syringae pv. syringae and $P$. syringae pv. pisi), to design more accurate detection and identification protocol, to identify a possible pathogenic specialization in $P$. syringae pv. syringae strains from pea, and to identify appropriate strains for disease resistance testing.

\section{ACKNOWLEDGMENTS}

This research was supported by projects ITACYL 2004/845 and GR113, from the Junta de Castilla y León, and RTA 2006-00077-00-00, from the INIA, as well as by an INIA personal Ph.D. fellowship to A. Martín-Sanz. We thank CFBP (France), NCPPB (United Kingdom), A. de Vicente (Universidad de Málaga, Spain), and J. L. Palomo (Centro Regional de Diagnóstico, Junta de Castilla y León, Spain) for providing bacterial strains; and two anonymous reviewers for their valuable comments and suggestions on the manuscript.

\section{LITERATURE CITED}

1. Almeida, N. F., Y. S., R. Cai, C. R. Clarke, C. E. Morris, N. W. Schaad, E. L. Schuenzel, G. H. Lacy, X. Sun, J. B. Jones, J. A. Castillo, C. T. Bull, S. Leman, D. S. Guttman, J. C. Setubal, and Vinatzer, B. A. 2010. PAMDB, a multilocus sequence typing and analysis database and website for plantassociated microbes. Phytopathology 100:208-215.

2. Arnold, D. L., Athey-Pollard, A., Gibbon, M. J., Taylor, J. D., and Vivian, A. 1996. Specific oligonucleotide primers for the identification of Pseudomonas syringae pv. pisi yield one of two possible DNA fragments by PCR amplification: evidence for phylogenetic divergence. Physiol. Mol. Plant Pathol. 49:233-245 (Erratum: 1997, 51:213).

3. Baltrus, D. A., Nishimura, M. T., Romanchuk, A., Chang, J. H., Mukhtar, M. S., Cherkis, K., Roach, J., Grant, S. R., Jones, C. D., and Dangl, J. L. 2011. Dynamic evolution of pathogenicity revealed by sequencing and comparative genomics of 19 Pseudomonas syringae isolates. PLoS Pathog. 7:e1002132.

4. Bradbury, J. F. 1986. Guide to Plant Pathogenic Bacteria, 1st ed. International Mycological Institute, Kew, UK.

5. Bull, C. T., Clarke, C. R., Cai, R., Vinatzer, B. A., Jardini, T. M., and Koike, S. T. 2011. Multilocus sequence typing of Pseudomonas syringae sensu lato confirms previously described genomospecies and permits rapid identification of $P$. syringae pv. coriandricola and $P$. syringae pv. apii causing bacterial leaf spot on parsley. Phytopathology 101:847-858.

6. Bull, C. T., De Boer, S. H., Denny, T. P., Firrao, G., Fischer-Le Saux, M., Saddler G. S., Scortichini, M., Stead, D. E., and Takikawa, Y. 2010. Comprehensive list of names of plant pathogenic bacteria, 1980-2007. J. Plant Pathol. 92:55-592.

7. Cai, R., Yan, S., Leman, S., and Vinatzer, B. A. 2011. Reconstructing host range evolution of bacterial plant pathogens using Pseudomonas syringae pv. tomato and its close relatives as a model. Infect. Genet. Evol. 11:17381751.

8. Dye, D. W., Bradbury, J. F., Goto, M., Hayward, A. C., Lelliott, R. A., and Schroth, M. N. 1980. International standards for naming pathovars of phytopathogenic bacteria and a list of pathovar names and pathotype strains. Rev. Plant Pathol. 59:153-168.

9. Felsenstein, J. 1989. PHYLIP—Phylogeny Inference Package (version 3.2). Cladistics 5:164-166

10. Ferrante, P., and Scortichini, M. 2010. Molecular and phenotypic features of Pseudomonas syringae pv. actinidiae isolated during recent epidemics of bacterial canker on yellow kiwifruit (Actinidiachinensis) in central Italy. Plant Pathol. 59:954-962.

11. Fondevilla, S., Martín-Sanz, A., Satovic, Z., Fernández-Romero, M. D., Rubiales, D., and Caminero, C. 2012. Identification of quantitative trait loci involved in resistance to Pseudomonas syringae pv. syringae in pea (Pisum sativum L.). Euphytica 186:805-812.

12. Gardan, L., Shafik, H., Belouin, S., Broch, R., Grimont, F., and Grimont, P. A. D. 1999. DNA relatedness among the pathovars of Pseudomonas syringae and description of Pseudomonas tremae sp. nov. and Pseudomonas cannabina sp. nov. (ex Sutic and Dowson 1959). Int. J. Syst. Bacteriol. 49:469-478

13. Hoitink, H. A., Hagedorn, D. J., and McCoy, E. 1967. Survival, transmission, and taxonomy of Pseudomonas syringae van Hall, the causal organism of bacterial brown spot of bean (Phaseolus vulgaris L.). Can. J. Microbiol. 14:437-441.

14. Hollaway, G. J., Bretag, T. W., and Price, T. V. 2007. The epidemiology and management of bacterial blight (Pseudomonas syringae pv. pisi) of 
field pea (Pisum sativum) in Australia: A review. Aust. J. Agric. Res. 58:1086-1099.

15. Hunter, P. J., and Taylor, J. D. 2006. Patterns of interaction between isolates of three pathovars of Pseudomonas syringae and accessions of a range of host and nonhost legume species. Plant Pathol. 55:46-53.

16. Hwang, M. S. H., Morgan, R. L., Sarkar, S. F., Wang, P. W., and Guttman, D. S. 2005. Phylogenetic characterization of virulence and resistance phenotypes of Pseudomonas syringae. Appl. Environ. Microbiol. 71:5182-5191.

17. Kennelly, M. M., Cazorla F. M., de Vicente, A., Ramos, C., and Sundin, G. W. 2007. Pseudomonas syringae diseases of fruit trees. Progress toward understanding and control. Plant Dis. 91:4-17.

18. Lawyer, A. S., and Chun, W. 2001. Foliar diseases caused by bacteria. Pages 22-24 in: Compendium of Pea Diseases, 2nd ed. J. M. Kraft and F. L. Pfleger, eds. American Phytopathological Society, St. Paul, MN.

19. Lelliot, R. A., Billing, E., and Hayward, A. C. 1966. A determinative scheme for the florescent plant pathogenic Pseudomonas. J. Appl. Bacteriol. 29:470-489.

20. Lindeberg, M., Cunnac, S., and Collmer, A. 2012. Pseudomonas syringae type III effector repertoires: Last words in endless arguments. Trends Microbiol. 4:199-208.

21. Louws, F. J., Dennos, W. F., Taylor, C., and Bruijn, F. J. 1994. Specific genomic fingerprints of phytopathogenic Xanthomonas and Pseudomonas pathovars and strains generated with repetitive sequences and PCR. Appl. Environ. Microbiol. 60:2286-2295.

22. Louws, F. J., Rademarker, J. L. W., and de Bruijn, F. J. 1999. The three Ds of PCR-based genomic analysis of phytobacteria: diversity, detection and diseases diagnosis. Annu. Rev. Phytopathol. 37:81-125.

23. Martín-Sanz, A., Palomo, J. L., and Caminero, C. 2009. First report of bacterial blight caused by Pseudomonas syringae pv. syringae on vetch in Spain. Plant Dis. 93:1348.

24. Martín-Sanz, A., Palomo, J. L., Pérez de la Vega, M., and Caminero, C. 2011. Identification of pathovars and races of Pseudomonas syringae, the main causal agent of bacterial diseases in pea in North-Central Spain, and the search for resistance. Eur. J. Plant Pathol. 129:57-69.

25. Martín-Sanz, A., Palomo, J. L., Pérez de la Vega, M., and Caminero, C. 2012. Characterization of Pseudomonas syringae pv. syringae isolates associated with bacterial blight in Lathyrus spp. and sources of resistance. Eur. J. Plant Pathol. 134:205-216.

26. Martín-Sanz, A., Pérez de la Vega, M., and Caminero, C. 2012. Resistance to Pseudomonas syringae in a collection of pea germplasm under field and controlled conditions. Plant Pathol. 61:375-387.

27. Martín-Sanz, A., Pérez de la Vega, M., Murillo, J., and Caminero, C.2012. Genetic, biochemical and pathogenic diversity of Pseudomonas syringae pv. pisi strains. Plant Pathol. 61:1063-1072.

28. O’Brien, H. E., Thakur, S., Gong, Y., Fung, P., Zhang, J., Yuan, L., Wang, P. W., Yong, C., Scortichini, M., and Guttman, D. S. 2012. Extensive remodeling of the Pseudomonas syringae pv. avellanae type III secretome associated with two independent host shifts onto hazelnut. BMC Microbiol. 12:141.
29. Oguiza, J. A., Rico, A., Rivas, L. A., Sutra, L., Vivian, A., and Murillo, J. 2004. Pseudomonas syringae pv. phaseolicola can be separated into two genetic lineages distinguishable by the possession of the phaseolotoxin biosynthetic cluster. Microbiology 150:473-482.

30. Parkinson, N., Bryant, R., Bew, J., and Elphinstone, J. 2010. Rapid phylogenetic identification of members of the Pseudomonas syringae species complex using the rpoD locus. Plant Pathol. 60:338-344.

31. Pritchard, J. K., Stephens, M., and Donnelly, P. 2000. Inference of population structure using multilocus genotype data. Genetics 155:945-959.

32. Rademarker, J. L., and de Bruijn, F. J. 1997. Characterization and classification of microbes by rep-PCR. Pages 151-171 in: DNA Markers: Protocols, Applications and Overview. G. Caetano-Anolles and P. M. Gresshoff, eds. John Wiley \& Sons, New York.

33. Richardson, H. J., and Hollaway, G. J. 2011. Bacterial blight caused by Pseudomonas syringae pv. syringae shown to be an important disease of field pea in south eastern Australia. Australas. Plant Pathol. 40:260-268.

34. Sarkar, S. F., Gordon, J. S., Martin, G. B., and Guttman, D. S. 2006. Comparative genomics of host-specific virulence in Pseudomonas syringae. Genetics 174:1041-1056.

35. Sarkar, S. F., and Guttman, D. S. 2004. Evolution of the core genome of Pseudomonas syringae, a highly clonal, endemic plant pathogen. Appl. Environ. Microbiol. 70:1999-2012.

36. Schaad, N. W., Jones, J. B., and Chun, W. 2001. Laboratory Guide for Identification of Plant Pathogenic Bacteria, 3rd ed. American Phytopathological Society, St. Paul, MN.

37. Scortichini, M., Marchesi, U., Dettori, M. T., and Rossi, M. P. 2003. Genetic diversity, presence of the $s y r B$ gene, host preference and virulence of Pseudomonas syringae pv. syringae strains from woody and herbaceous host plants. Plant Pathol. 52:277-286.

38. Sorensen, K. N., Kim, K. H., and Takemoto, J. Y. 1998. PCR detection of cyclic lipodepsinonapeptide-producing Pseudomonas syringae pv. syringae and similarity of strains. Appl. Environ. Microbiol. 64:226-230.

39. Studholme, D. J. 2011. Application of high-throughput genome sequencing to intrapathovar variation in Pseudomonas syringae. Mol. Plant Pathol. 12:829-838.

40. Tamura, K. 1992. Estimation of the number of nucleotide substitutions when there are strong transition-transversion and $\mathrm{G}+\mathrm{C}$ content biases. Mol. Biol. Evol. 9:678-687.

41. Tamura, K., Peterson, D., Peterson, N., Stecher, G., Nei, M., and Kumar, S. 2011. MEGA5: molecular evolutionary genetics analysis using maximum likelihood, evolutionary distance, and maximum parsimony methods. Mol. Biol. Evol. 28:2731-2739.

42. Vinatzer, B. A., Gail, M. T., Lee, M., Jelenska, J., Hotton, S., Fairfax, K., Jenrette, J., and Greenberg, J. T. 2006. The type III effector repertoire of Pseudomonas syringae pv. syringae B728a and its role in survival and disease on host and non-host plants. Mol. Microbiol. 62:26-44.

43. Vivian, A., and Arnold, D. L. 2000. Bacterial effector genes and their role in host-pathogen interactions. J. Plant Pathol. 82:163-178.

44. Young, J. M. 2010. Taxonomy of Pseudomonas syringae. J. Plant Pathol. 92:S1.5-S1.14 